\title{
CARACTERÍSTICAS DETERMINANTES ENTRE PORTADORES DE HANSENÍASE EM UMA ÁREA HIPERENDÊMICA
}

\author{
DETERMINANT CHARACTERISTICS BETWEEN LEPROSY PATIENTS IN
} HYPERENDEMIC AREA IN THE SOUTH OF TOCANTINS Julliany Lopes Dias', Gracielle Mara Silva Godoy², Ricardo Saraiva Aguiar ${ }^{3}$, Giselle Pinheiro Lima Aires Gomes ${ }^{4}$

\author{
'Enfermeira do Hospital Regional de Gurupi - Gurupi (TO), Brasil. Especialista em Unidade de Terapia Intensiva - Goiânia (GO), Brasil. \\ ${ }^{2}$ Enfermeira. Especialista em Unidade de Terapia Intensiva - Goiânia (GO), Brasil. \\ ${ }^{3}$ Enfermeiro do Núcleo de Saúde da Criança, Secretaria de Saúde do Distrito Federal - Brasília (DF), Brasil. Especialista em Saúde da Família - \\ Brasília (DF), Brasil. \\ ${ }^{4}$ Enfermeira. Professora no curso de Enfermagem da Universidade Federal do Tocantins - Palmas (TO), Brasil. Mestre em Ciências da \\ Motricidade Humana pela Universidade Castelo Branco - Rio de Janeiro (RJ), Brasil.
}

Data de entrada do artigo: $24 / 01 / 2013$

Data de aceite do artigo: 15/04/2013

\section{RESUMO}

Introdução: A hanseníase é uma doença infectocontagiosa de evolução lenta que se manifesta principalmente por lesões na pele e nos nervos periféricos. Objetivo: Identificar as características determinantes em portadores de hanseníase e os fatores para o desenvolvimento da doença em uma área hiperendêmica. Materiais e Métodos: Trata-se de um estudo descritivo exploratório. Participaram da pesquisa 25 indivíduos do município de Gurupi (TO), cujos perfis foram traçados por meio de informações pertinentes ao nível socioeconômico, ao estado nutricional, às condições de moradia e ao histórico de doenças pessoais e familiares. Resultados: Os resultados coletados foram avaliados descritivamente e confrontados com dados de pesquisas anteriores. Conclusões: Tornou-se possível estabelecer que a associação das variáveis encontradas, tais como baixa escolaridade e renda familiar, nível nutricional inadequado, más condições de moradia e higiênico-sanitárias é um fator que propicia a sobrevida do bacilo e sua disseminação, bem como torna os indivíduos que vivem essa forma desfavorável de vida mais suscetíveis a adoecer, resultando na hiperendemicidade do município.

Palavras-chave: hanseníase; hiperendemia; ações de Saúde.

\section{ABSTRACT}

Introduction: Leprosy is a contagious infectious disease of slow evolution, manifested primarily by skin lesions and peripheral nerve. Objective: Identify determinant features of leprosy patients and the factors for the development of disease in a hyper endemic area. Material and Methods: This is a descriptive exploratory study. Twenty-five individuals of the city of Gurupi (TO) have participated of the research, whose profile was traced through pertinent information to the socioeconomic level, nutritional status, living conditions and personal history of disease and family. Results: The collected results were analyzed descriptively and compared with data from previous research Conclusion: It was possible to establish that the association of variables, such as low education and income, inadequate nutritional level, bad conditions of housing and hygienically-sanitary is a factor that promotes the survival of the bacillus and its dissemination, and makes individuals who lives this form of adverse life to be more susceptible to illness, resulting in hyperendemicity the municipality.

Keywords: leprosy; hyper endemic; health actions. 


\section{INTRODUÇÃO}

A hanseníase, conhecida desde a Antiguidade como lepra, é uma doença infectocontagiosa de evolução lenta que se manifesta principalmente por lesões na pele e nos nervos periféricos. Tais manifestações são causadas pelo Mycobacterium leprae, mais conhecido como bacilo de Hansen, bactéria de alta infectividade e baixa patogenicidade ${ }^{1-3}$.

A característica mais preocupante da doença consiste no comprometimento dos nervos periféricos, podendo evoluir para deformidades e desencadear problemas como a limitação da vida social e a redução da capacidade de trabalho, além de problemas psicológicos resultantes do estigma e do preconceito que ainda sofrem os portadores. No entanto, é importante ressaltar que a hanseníase tem cura e, se diagnosticada precocemente, as incapacidades podem ser evitadas ou reduzidas.

Desde a década de 1980, um esforço global tem sido empregado para a eliminação desse problema de saúde pública. Porém, alguns países da faixa intertropical, como Índia, Brasil, Myanmar, Madagascar, Nepal e Moçambique, ainda se mostram endêmicos para a doença ${ }^{4}$. Essa perspectiva evidencia a elevada incidência da hanseníase em países subdesenvolvidos, que encontra como principal subsídio as condições individuais e socioeconômicas dessas populações.

O Brasil é o segundo país no mundo com o maior número de casos, perdendo apenas para a Índia. Esse dado torna a doença um grave problema de saúde pública apesar dos esforços para sua erradicação4.

Estudos sobre a situação epidemiológica da doença no Brasil realizados pelo Ministério da Saúde revelam que, entre 2001 e 2008, foram diagnosticados 370.162 casos novos, concentrados em 1.173 municípios brasileiros, principalmente nas regiões Norte, Centro-Oeste e Nordeste, que registraram $53,5 \%$ das novas ocorrências detectadas entre 2005 e $2007^{5}$.

Segundo dados do Sistema de Informação de Agravos de Notificação (SINAN), cedidos pela Secretaria Municipal de Saúde de Gurupi (TO), no ano de 2009 foi revelada a notificação de 116 novos casos na cidade supracitada, o que corresponde a um índice de detecção de 15,6 casos/10.000 habitantes e caracteriza a área como hiperendêmica, haja vista que o índice classifica no parâmetro hiperendêmico valores de detecção igual ou superior a 4 casos para cada 10.000 habitantes $^{2}$.

Assim, a hiperendemicidade da hanseníase no município de Gurupi (TO), a presença de poucos estudos sobre a temática no município e a incerteza do peso que variáveis como moradia, estado nutricional, infecções concomitantes e prévias, bem como fatores genéticos exercem na distribuição da doença perfazem a problemática deste estudo, que buscou identificar quais dessas condições comuns aos hansenianos podem ser determinantes para o desenvolvimento da doença.

Dessa forma, este estudo tem por objetivo identificar as características determinantes em portadores de hanseníase e os fatores para o desenvolvimento da doença em uma área hiperendêmica.

\section{MATERIAIS E MÉTODOS}

Trata-se de um estudo descritivo exploratório que teve por objetivo identificar as características determinantes aos hansenianos de uma área hiperendêmica na região Sul do Estado do Tocantins e investigar quais dessas condições podem ser determinantes para o desenvolvimento da doença.

O estudo teve como campo o domicílio dos pacientes em tratamento situados na zona urbana do município de Gurupi (TO). O endereço das residências foi cedido pelas enfermeiras das Unidades Básicas de Saúde (UBS) com autorização prévia do secretário municipal de saúde e dos pacientes.

Participaram do estudo 25 indivíduos, que atenderam aos seguintes critérios de inclusão: pacientes notificados em 2009 e 2010, ativos no tratamento, maiores de 18 anos, residentes na área urbana de Gurupi (TO) e que aceitaram participar do estudo após assinar o Termo de Consentimento Livre e Esclarecido (TCLE).

Como instrumento para a coleta dos dados, foi realizada uma entrevista semiestruturada. A entrevista seguiu o seguinte roteiro, dividido em duas partes: parte I (questionário socioeconômico) e parte II (investigação de doenças prévias e concomitantes, coleta de informações pertinentes ao estado nutricional, bem como a averiguação das condições de moradia, com posterior mensuração da temperatura e umidade do ambiente).

Como critérios para a análise da ventilação e iluminação natural dos domicílios, utilizaram-se os parâmetros: número de janelas por cômodo, dimensão da janela ( $\geq a 1 \times 1,20 \mathrm{~m}$ ) e tempo de permanência diária das janelas abertas no período diurno ( $\geq 4 \mathrm{~h}$ ) como indício de boa ventilação e iluminação. Considerou-se como regular o domicílio que atendesse a dois dos critérios supracitados e ruim aquele que não atendesse a nenhum ou apenas um desses critérios.

Na avaliação da temperatura e da umidade do ambiente foi utilizado um termo-higrômetro da marca INCOTERM ${ }^{\circledR}$, capaz de mensurar simultaneamente a temperatura e a umidade. Esses valores foram mensurados nos cômodos de maior aglomeração da residência (sala e quarto). 
O nível de limpeza dos domicílios visitados foi investigado por meio de análise observacional e classificado em bom, regular e ruim, de acordo com a presença ou não de sujidades aparentes, acúmulo ou não de lixo, presença ou ausência de insetos, poeira e odores.

Este estudo não teve conflitos de interesse e foi desenvolvido respeitando às normas estabelecidas na Resolução no 196/96 do Conselho Nacional de Saúde (CNS) de 10 de outubro de 1996, que se refere à realização de pesquisa em seres humanos, com o consentimento prévio da Secretaria Municipal de Saúde de Gurupi (TO), após aprovação no Comitê de Ética e Pesquisa do Centro Universitário UNIRG (processo n 116/2010).

\section{RESULTADOS E DISCUSSÃO}

Com relação à idade dos portadores de hanseníase, encontrou-se maior prevalência da doença na faixa etária de 69 a 78 anos (28\%) e a menor incidência entre os entrevistados estava na faixa etária de 60 a 69 anos (8\%) (Tabela 1).

Os resultados obtidos assemelham-se com outro estudo em que o número de casos aumentou com o progredir da idade, com predominância no grupo de 35 a 64 anos (39\%), seguidos da faixa etária de 18 a 34 anos (34\%). No entanto, essa pesquisa é divergente com relação à faixa etária superior a 65 anos,

Tabela 1: Perfil dos pacientes portadores de hanseníase. Gurupi (TO), 2010.

\begin{tabular}{lcc}
\hline Sexo & $\mathbf{n}$ & $\mathbf{\%}$ \\
\hline Masculino & 11 & 44 \\
\hline Feminino & 14 & 56 \\
\hline Total & 25 & 100 \\
\hline Idade & & \\
\hline $24-33$ anos & 4 & 16 \\
\hline $33-42$ anos & 3 & 12 \\
\hline $42-51$ anos & 5 & 20 \\
\hline $51-60$ anos & 4 & 16 \\
\hline $60-69$ anos & 2 & 8 \\
\hline $69-78$ anos & 7 & 28 \\
\hline Total & 25 & 100 \\
\hline Escolaridade & & \\
\hline Fundamental & 13 & 65 \\
\hline Ensino médio & 6 & 30 \\
\hline Ensino superior & 1 & 5 \\
\hline Total & 25 & 100 \\
\hline Renda familiar (salário) & & \\
\hline $0-1$ & 1 & 4 \\
\hline $1-2$ & 7 & 28 \\
\hline $2-3$ & 11 & 44 \\
\hline $3-4$ & 1 & 4 \\
\hline $4-5$ & 5 & 20 \\
\hline Total & 25 & 100 \\
\hline & &
\end{tabular}

na qual houve a menor incidência de portadores, pois no presente estudo esse grupo foi o mais acometido ${ }^{6}$.

No tocante ao gênero, prevaleceram os portadores do sexo feminino, com índice de $56 \%$ dos casos (Tabela 1).

Alguns fatores podem justificar o fato de a taxa de detecção ser maior entre as mulheres, tais como maior preocupação com o corpo e a estética e facilidade de acesso das mulheres às UBS em função de programas (pré-natal, planejamento familiar, programa cérvico-uterino e de câncer de mama), ao contrário dos homens, que ainda não dispõem de programas estruturados de prevenção e acompanhamento. Além disso, pode-se elencar a mudança na participação das mulheres no mercado de trabalho, levando a uma maior exposição destas à comunidade e a um aumento de suas possibilidades de contato com portadores da doença.

Dessa forma, se os homens tivessem a mesma oportunidade de diagnóstico que as mulheres, possivelmente haveriam percentuais iguais de distribuição e, logo, maiores taxas de detecção. Assim, esses fatores podem determinar diferenciais entre os gêneros quanto a incidência, oportunidade do diagnóstico (precoce ou tardio) e, em consequência, instalação de incapacidades, que se evidenciam entre os homens ${ }^{7}$.

Considerando o nível de instrução dos portadores de hanseníase entrevistados, verificou-se que, apesar de em menor proporção, os analfabetos constituem $20 \%$ dos indivíduos pesquisados, média acima da taxa nacional de analfabetismo divulgada pela Pesquisa Nacional por Amostra de Domicílios de 2009 (PNAD), que é de 9,7\% ${ }^{8}$.

Dos alfabetizados, a maioria (65\%) concluiu apenas o nível fundamental de ensino, resultado similar ao identificado em outro estudo ${ }^{9}$, no qual a maioria dos entrevistados $(55,6 \%)$, tinham também apenas nível fundamental.

Tais dados reforçam a ideia de que o baixo nível de escolaridade influencia na disseminação da doença, uma vez que os indivíduos pouco conhecem sobre ela, tampouco sobre de que forma prevenir.

De acordo com a tabela 1, 32\% apresentam renda menor ou igual a DOIS salários mínimos.

Quando questionados sobre às profissões anteriores, permaneceu a incidência das profissões de baixa remuneração e de menor exigência de escolaridade, com a prevalência das seguintes ocupações: doméstica (32\%), agricultor e pedreiro (ambos com $12 \%$ ).

Os dados coletados demonstram ainda um predomínio de maus hábitos alimentares, resultando em dietas desequilibradas, arraigadas de excessos de grãos, açúcar e gorduras e deficientes em nutrientes presentes em verduras e frutas, como demonstra a Tabela 2. 
Tabela 2: Tipo de dieta dos pacientes portadores de hanseníase. Gurupi (TO), 2010.

\begin{tabular}{lcc}
\hline Rica em vegetais & $\mathbf{n}$ & $\mathbf{\%}$ \\
\hline Sim & 11 & 44 \\
\hline Não & 14 & 56 \\
\hline Total & 25 & 100 \\
\hline Rica em frutas & & \\
\hline Sim & 16 & 64 \\
\hline Não & 9 & 36 \\
\hline Total & 25 & 100 \\
\hline Rica em grãos & & \\
\hline Sim & 23 & 92 \\
\hline Não & 2 & 8 \\
\hline Total & 25 & 100 \\
\hline Moderada em gordura e açúcar & & \\
\hline Sim & 12 & 48 \\
\hline Não & 13 & 52 \\
\hline Total & 25 & 100 \\
\hline
\end{tabular}

Tabela 3: Características dos domicílios dos pacientes portadores de hanseníase. Gurupi (TO), 2010.

\begin{tabular}{|c|c|c|}
\hline Ventilação e iluminação & $\mathbf{n}$ & $\%$ \\
\hline Boa & 10 & 40 \\
\hline Regular & 4 & 16 \\
\hline Ruim & 11 & 44 \\
\hline Total & 25 & 100 \\
\hline \multicolumn{3}{|l|}{ Limpeza } \\
\hline Boa & 10 & 10 \\
\hline Regular & 11 & 44 \\
\hline Ruim & 4 & 16 \\
\hline Total & 25 & 100 \\
\hline \multicolumn{3}{|l|}{ Temperatura } \\
\hline $28,5-30,5$ & 3 & 12 \\
\hline $30,5-32,5$ & 1 & 4 \\
\hline $32,5-34,5$ & 7 & 28 \\
\hline $34,5-36,5$ & 8 & 32 \\
\hline $36,5-38,6$ & 6 & 24 \\
\hline Total & 25 & 100 \\
\hline \multicolumn{3}{|l|}{ Umidade } \\
\hline $22,0-31,6$ & 6 & 24 \\
\hline $31,6-41,2$ & 9 & 36 \\
\hline $41,2-50,8$ & 3 & 12 \\
\hline $51,8-60,4$ & 5 & 20 \\
\hline $60,4-70,0$ & 2 & 8 \\
\hline Total & 25 & 100 \\
\hline \multicolumn{3}{|l|}{ Água tratada } \\
\hline Sim & 24 & 96 \\
\hline Não & 1 & 4 \\
\hline Total & 25 & 100 \\
\hline \multicolumn{3}{|l|}{ Destino dos dejetos } \\
\hline Fossa negra & 14 & 56 \\
\hline Fossa séptica & 7 & 28 \\
\hline Esgoto & 4 & 16 \\
\hline Total & 25 & 100 \\
\hline \multicolumn{3}{|l|}{ Frequência da coleta de lixo } \\
\hline Diária & 11 & 44 \\
\hline 2 a 3 vezes por semana & 14 & 56 \\
\hline Total & 25 & 100 \\
\hline
\end{tabular}

Quando indagados sobre outros alimentos de uso frequente, mostrou-se demasiadamente elevada a ingestão de carnes de animais silvestres, destacando-se tatu, paca, anta, capivara e veado. Dentre os animais citados é de extrema relevância ressaltar o tatu, haja vista que é considerado hospedeiro intermediário do Mycobacterium leprae, bacilo causador da hanseníase.

Em estudo sobre os aspectos epidemiológicos da transmissão da hanseníase em relação ao contato com o tatu, obteve-se, com as entrevistas de 136 hansenianos e ex-hansenianos, o relato de 120 individuos que consumiram carne de tatu na dieta e posteriormente desenvolveram a doença ${ }^{10}$.

No estudo houve um alto índice de aglomeração de pessoas nas residências dos hansenianos, como mostra o Gráfico 1, visto que, ao se dividir o número de moradores por cômodo, a proporção na maioria dos casos foi de menos de um cômodo por morador.

De acordo com os critérios utilizados na metodologia deste estudo, para classificar a qualidade da ventilação e iluminação natural das residências, verificou-se que em $44 \%$ das moradias a ventilação e iluminação era ineficaz, $16 \%$, regular e $40 \%$, boa (Tabela 3 ).

Houve a predominância de uma janela por cômodo, no entanto, a maioria das janelas tinha dimensões pequenas e permanecia fechada por um longo período (Gráfico 2). Esses fatores minimizam o potencial de ventilação e iluminação no interior das residências, favorecendo a sobrevivência do bacilo por maior tempo, bem como a exposição dos indivíduos ao patógeno, o que pode colaborar para a disseminação da doença.

O Mycobacterium leprae pode sobreviver, durante meses, fora do corpo humano e em condições favoráveis de umidade. Assim, solos úmidos, baixas temperaturas e elevada umidade ambiental favorecem a sobrevivência do bacilo 4 .

Quando analisadas as umidades e temperaturas decorrentes de mensuração no interior dos domicílios, constatou-se o predomínio de altas temperaturas, entre 34,5 a $36,5^{\circ} \mathrm{C}$, e baixa umidade, com índice pico de 31,3 a $41,2 \%$ (Tabela 3).

Entretanto, esses dados são pontuais e é importante a ressalva de que o município encontra-se em região onde as estações climáticas são apenas duas bem definidas, seca e chuvosa, e que a chuvosa se apresenta maior que a seca. Em contrapartida, o período no qual o presente estudo foi realizado compreende os meses setembro e outubro, os quais perfazem a temporada em que as precipitações pluviométricas ocorrem em menor quantidade, fato que justifica a baixa umidade na época. 


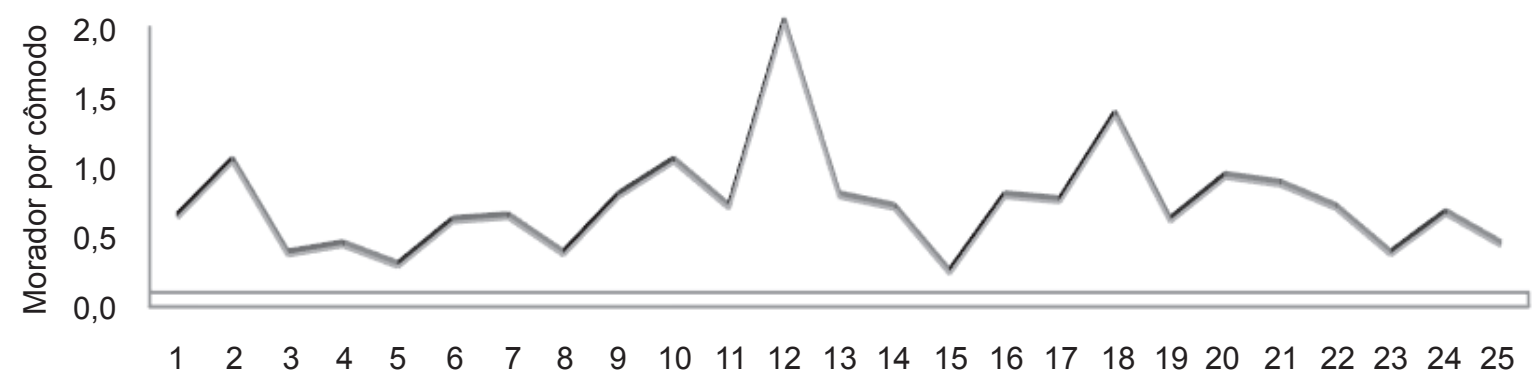

Gráfico 1: Quantidade de morador por cômodo na residência dos portadores de hanseníase. Gurupi (TO), 2010.

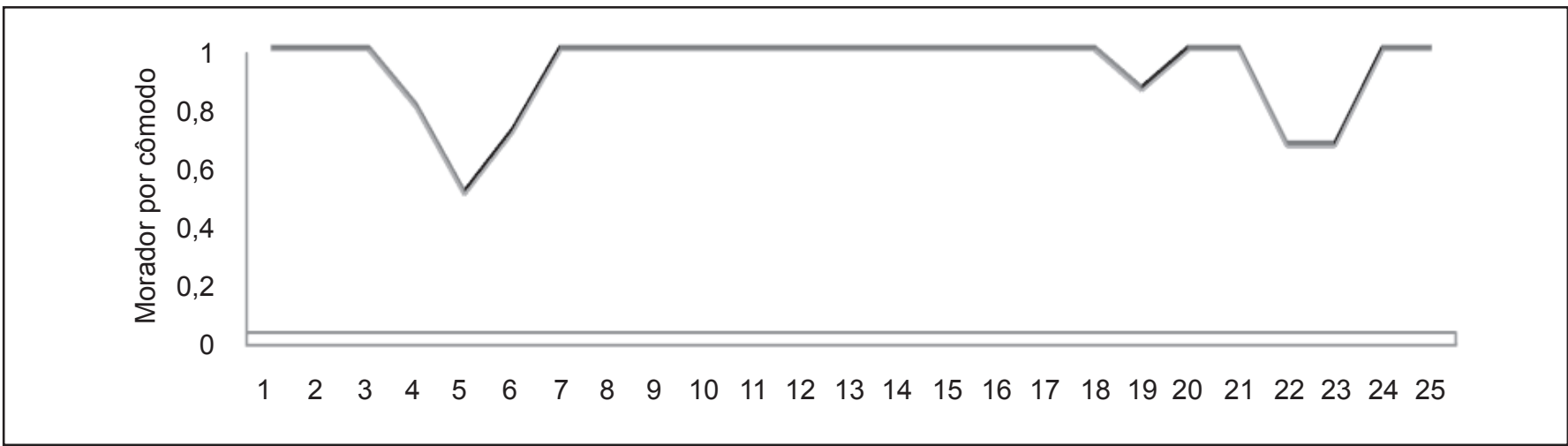

Gráfico 2: Quantidade de janelas por cômodo na casa dos portadores de hanseníase. Gurupi (TO), 2010.

O Mycobacterium leprae permanece viável por até 36 horas no meio ambiente, ou por aproximadamente 9 dias em temperaturas de $36,7^{\circ} \mathrm{C}$ e umidade média de $77,6 \%$. Nas regiões tropicais, as secreções nasais de pacientes multibacilares mantêm-se viáveis por até 9 dias e, no solo úmido, em temperatura ambiente, por até 46 dias ${ }^{11}$.

Mas, além de microambientes favoráveis a existência e sobrevivência do patógeno, outros fatores propícios à transmissão ou mesmo evolução clínica da hanseníase devem ser analisados ${ }^{4}$.

Nos domicílios dos entrevistados, em relação à limpeza, constatou-se o predomínio de $44 \%$ das residências com nível regular, $40 \%$ com bom aspecto de higienização e $16 \%$ classificadas como ruim.

Quanto ao uso de água tratada nos domicílios visitados, observou-se que $96 \%$ recebem tratamento e $4 \%$ não empregavam nenhum mecanismo de tratamento. Verificou-se ainda que em $40 \%$ das residências a água utilizada era proveniente de poços.

Sobre o destino dos dejetos, observou-se que, nos domicílios dos pacientes estudados, a maioria (56\%) tinha fossa negra e a minoria (16\%) tinha acesso ao esgoto.

Em média, $48 \%$ da população das microrregiões que compõem a Amazônia brasileira residem em domicílios com fossa rudimentar, sendo esse termo empregado quando o banheiro ou sanitário é ligado a uma fossa rústica (fossa negra, poço ou buraco, por exemplo) ${ }^{11}$.

O alto índice de residentes em domicílios com fossa negra configura-se como uma característica comum na formação da cidade, uma vez que a ocupação desordenada dificultou a estruturação sanitária.

A totalidade das famílias estudadas dispunha de acesso à coleta de lixo. Porém, a coleta diária é benefício de somente $44 \%$ dos entrevistados, enquanto os $56 \%$ restantes dispõem apenas de 2 a 3 coletas semanais.

Com base nos aspectos apresentados, tornam-se necessárias e urgentes medidas que revelem o lado oculto do "iceberg epidemiológico" da doença com a finalidade de se reduzir a morbidade da patologia na população.

Dessa forma, o desafio para a redução da prevalência da hiperendemia hansênica persiste. Nesse sentido, este estudo reforça a necessidade da realização de pesquisas regionais, para que seja conhecida a distribuição da doença localmente. Faz-se necessário a implementação de estratégias que intensifiquem a busca ativa, a atualização periódica do banco de dados e as medidas profiláticas preconizadas pelo Ministério 
da Saúde, tais como o exame de contatos e a vacinação do BCG-ID.

Além disso, faz-se necessária a promoção da educação continuada da equipe multiprofissional da atenção primária e a realização de ações educativas voltadas para a população com o objetivo de orientar o doente, a família e a comunidade sobre aspectos clínicos, epidemiológicos e terapêuticos a fim de reduzir o estigma e preconceito que ainda cercam a doença.

\section{CONCLUSÕES}

A hanseníase por ser uma doença milenar e ter aspectos fundamentais de sua epidemiologia ainda hoje não elucidados desperta o interesse em diagnosticar fatores que sejam determinantes para o desenvolvimento da doença. Diante disso, este estudo teve por escopo identificar quais condições comuns que tinham os portadores de hanseníase do município de Gurupi podem ter influenciado o adoecimento deles.

Percebeu-se que ao analisar as variáveis, algumas características associadas podem propiciar uma sobrevida maior do bacilo de Hansen. Nesse sentido, a baixa condição socioeconômica, a baixa escolaridade, o estado nutricional inadequado, os maus hábitos alimentares e higiênico-sanitários dos entrevistados de forma associada pode levar a uma maior disseminação da bactéria, bem como pode tornar os indivíduos mais suscetíveis ao adoecimento.

Portanto, este estudo sugere que sejam realizadas ações de conscientização dos enfermeiros e demais profissionais responsáveis pela saúde desses indivíduos no sentido de facilitar que pesquisas como esta sejam realizadas para que, assim, possam ser identificados fatores definidores da hanseníase, possibilitando a redução da incidência ou erradicação da doença no município.

\section{REFERÊNCIAS}

1. Araújo MG. Hanseníase no Brasil. Rev Soc Bras Med Trop. 2003; 36(3):373-382.

2. Ministério da Saúde (Brasil). Secretaria de Políticas de Saúde. Departamento de atenção Básica. Guia para o controle da hanseníase. Brasília: Ministério da Saúde; 2002.

3. Eidt LM. Breve história da hanseníase: sua expansão do mundo para as Américas, Brasil e o Rio Grande do Sul e sua trajetória na saúde pública brasileira. Saúde Soc. 2004; 13(2):76-88.

4. Magalhães MCC, Rojas LI. Diferenciação territorial da hanseníase no Brasil. Epidemiol Serv Saúde. 2007; 16(2):75-84.

5. Ministério da Saúde (Brasil). Secretaria de Vigilância em Saúde. Departamento de Vigilância Epidemiológica. Programa Nacional de Controle de Hanseníase. Vigilância em Saúde: situação epidemiológica da hanseníase no Brasil - 2008. Brasília: Ministério da Saúde; 2008.

6. Hinrichsen SI, Pinheiro MRS, Juca MB, Rolim H, Danda GJN, Danda DMR. Aspectos epidemiológicos da hanseníase na cidade de Recife, PE em 2002. An Bras Dermatol. 2004 jul./ago.; 79 (4):413-421.

7. Lana FCF, Davi RFL, Lanza FM, Amaral EP. Detecção da hanseníase e índice de desenvolvimento humano dos municípios de Minas Gerais, Brasil. Rev Eletr Enf. 2009; 11(3):539-544.

8. Ministério da Fazenda (Brasil). Secretaria de Política Econômica. Pesquisa Nacional por Amostra de Domicílios (PNAD) 2009 - IBGE. Brasília: Ministério da Fazenda; 2009.

9. Santos AS, Castro DS, Falqueto A. Fatores de risco para transmissão da hanseníase. Rev Bras de Enf. 2008;61(esp):738-743.

10. Deps PD, Faria LV, Gonçalves VC, Silva DA, Ventura CG, Zandonade E. Aspectos epidemiológicos da transmissão da hanseníase em relação à exposição ao tatu. Hansen Int. 2003; 28(2):138-44.

11. Silva DRX, Ignotti E, Souza-Santos R, Hacon SS. Hanseníase, condições sociais e desmatamento na amazônia brasileira. Rev Panam Salud Publica. 2010; 27(4):268-275.

\section{Endereços para correspondência:}

Julliany Lopes Dias

jullianyld@gmail.com>

Gracielle Mara Silva Godoy

gragracinha_@hotmail.com
Ricardo Saraiva Aguiar

rick-aguiar@hotmail.com

Giselle Pinheiro Lima Aires Gomes

gi_pinheiro@hotmail.com 